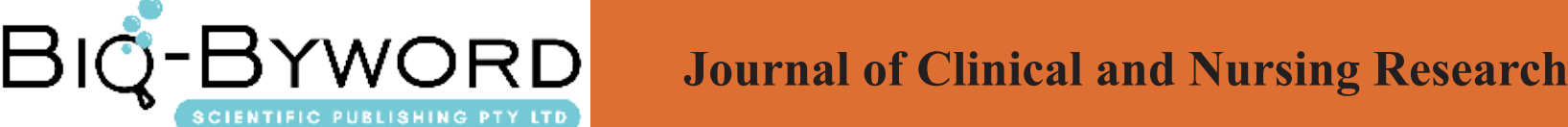

\section{Risk Assessment and Countermeasures of Fall in Elderly Patients}

\section{Li Hongjun}

Department of Hematology, South Building, PLA General Hospital, Beijing 100853, China

\section{ARTICLE INFO}

Article history:

Published online: 15th July, 2017

Key words:

The elderly patient

fall risk assessment

Countermeasures

\section{ABSTRACT}

Abstract: Objective: To investigate the risk factors of falls in elderly patientsv and put forward feasible measures. Methods: A total of 107 elderly patients were enrolled in our department from May 2014 to May 2015 to assess the risk factors for their fall. Results: The risk factors of falls in elderly patients were: body function degradation, disease factors, mental state change, medication, and environmental factors. Conclusion: There are many factors that lead to the fall of elderly patients, to take targeted measures can effectively prevent the occurrence of falls.

\section{Introduction}

Elderly patients with physiological function degenerative changes and activity inconvenience are more likely to fall. Accurate assessment of elderly patients during hospitalization falls risk factors to find the appropriate nursing measures for the elderly patients to create a safe environment has become an important issue in the elderly ward care work. Now in May 2014 - May 2015, our hospital in elderly patients fall risk factors and countermeasures are summarized as follows:

\section{General information}

From May 2014 to May 2015, we have a total of 107 elderly patients aged over 65 in our department, of whom $65-85$ are 79 , accounting for $73.8 \%$, and 18 are over 18 years old, accounting for $26.2 \%$.

\section{Risk assessment of falls}

\subsection{Body function degradation}

The elderly feel the information will be simplified, weakened, the reaction time will increase. The environment cannot change the timely changes in the appropriate action and fall. Studies have shown that both the decrease in visual acuity and the degradation of stereoscopic perception will increase the risk of the elderly by 6 times ${ }^{[1]}$. It has also been pointed out that the ankle input orientation sensation is more important in the prevention of falls than in the visual reference ${ }^{[2]}$.

\subsection{Disease factors}

Elderly patients often due to cardiovascular disease, neurological diseases and other chronic diseases admitted to the hospital, the disease itself will cause 
weakness, dizziness, combined with the hospital environment unfamiliar, treatment of stress can exacerbate the disease, the possibility of falling accidents will increase greatly.

\subsection{Mental state changes}

China's population over 60 years of age the prevalence of senile dementia was $0.75 \%-4.69 \%{ }^{[3]}$, such patients due to cognitive and memory loss, prone to lethargy, blurred, abnormal orientation, etc., there are a lot of falls hidden.

\subsection{Drug safety factors}

Most of the elderly need long-term use of a variety of drugs, drug metabolism is weakened, prone to drug accumulation and adverse drug reactions ${ }^{[4]}$.

\subsection{Environmental factors}

The safety of the elderly's life or not by its lifestyle and living environment factors. Studies have shown that over the age of $65,51 \%$ of the fall occurred with environmental factors, these environmental factors, including the elderly surrounding the environment is dangerous, disorder and the elderly can adapt to the environment.

\section{Response measures}

\subsection{Assess safety and strengthen mission}

Ensure that the patient is familiar with the ward environment, layout and facilities, and inquire about the history. According to the "falls risk factor score" to assess the existence of the risk of falls in the elderly patients, put forward preventive measures, easy to fall in the back of the patient suspended warning signs, to strengthen the anti-fall knowledge of the mission, so that patients with anti-fall concerns and methods.

\subsection{Environmental safety mainly for prevention}

$33 \%$ of elderly patients fall in the night, night more common on the bathroom fell most ${ }^{[5]}$. Therefore, the ward must be to maintain effective lighting, do not place too much debris, bathroom and bathroom are equipped with anti-skid mats, toilet with a handrail and call device. Ward has just dragged the wet surface should be placed eye-catching anti-fall logo, ward corridor with handrails to facilitate the patient walking or walking.

\subsection{Drug safety cannot be ignored}

Should explain in detail to the patient adverse reactions and precautions, sleep sedation should be taken in bed before bed, after taking no longer activities, antihypertensive drugs should not be replaced immediately after taking the position, hypoglycemic drugs must be strictly in accordance with the time to take, To prevent the occurrence of falling accidents.

\section{References}

[1] Ma Jingdong, Liu Yuxian. Risk factors for the Occurrence of Fractures in the Elderly [J]. Foreign Medical Social Medicine, 2011,19 (6): 70

[2] Xiao Chunmei, Zhou Jialin, Li Yang, et al. Research Progress on the Factors Related to the Fall of the Elderly [J]. Chinese Journal of Clinical Rehabilitation, 2012,6 (4): 1014

[3] He Wei Psychiatry [M] Beijing: People's Health Publishing House 2002: 58

[4] Wang Xiaoyuan, Hao Jianling, etc. to improve the elderly patients with oral medication safety care measures [J] Journal of Nursing Journal 2007.24 (7): 22-25

[5] Ma Wei. Special Care Ward Patients Fall Effective Prevention $[\mathrm{J}]$ Foreign Medical Nursing Volume 1997.16 (5): 20 